\title{
Carlos FAUSTO, Art Effects. Image, Agency, and Ritual in
} Amazonia

\section{Cyril Menta}

\section{OpenEdition}

\section{Journals}

Édition électronique

URL : https://journals.openedition.org/jsa/19719

DOI : 10.4000/jsa. 19719

ISSN : 1957-7842

\section{Éditeur}

Société des américanistes

\section{Édition imprimée}

Date de publication : 30 septembre 2021

Pagination : 245-249

ISSN : 0037-9174

\section{Référence électronique}

Cyril Menta, "Carlos fausto, Art Effects. Image, Agency, and Ritual in Amazonia », Journal de la Société des américanistes [En ligne], 107-1 | 2021, mis en ligne le 30 septembre 2021, consulté le 03 septembre 2022. URL : http://journals.openedition.org/jsa/19719; DOI : https://doi.org/10.4000/jsa. 19719

Ce document a été généré automatiquement le 3 septembre 2022

Tous droits réservés 


\title{
Carlos FAUSTO, Art Effects. Image, Agency, and Ritual in Amazonia
}

\author{
Cyril Menta
}

\section{RÉFÉRENCE}

Carlos FAUSTO, Art Effects. Image, Agency, and Ritual in Amazonia, traduit du portugais par David Rogers, University of Nebraska Press, Lincoln, 420 p., 2020, bibliogr., index, 11 ill. (en noir et blanc), 45 photos (en noir et blanc), 5 cartes, 7 tabl.

1 Carlos Fausto travaille depuis plus de vingt ans auprès des Kuikuro, groupe de langue caribe vivant dans le Haut-Xingu. Il avait au préalable mené des recherches auprès des Parakanã, groupe tupi-guarani de l'actuel État du Pará. Une dissemblance tangible entre ces deux terrains est à l'origine du présent ouvrage: chez les Kuikuro, pas un jour ne semble se passer sans qu'une activité cérémonielle ait lieu ; chez les Parakanã, de telles manifestations sont rares. De l'incompréhension initiale de l'auteur face à la possession et à l'usage d'artefacts rituels, de nombreuses réflexions et hypothèses ont germé sur les relations entre personnes et choses, ainsi que sur l'agentivité des images et des artefacts, sur la « présence » induite (dans les situations rituelles) par le maniement d'artefacts, sur l'élaboration de graphismes, ou encore, sur l'énonciation de chants.

2 Ce livre est une étude anthropologique d'expressions artistiques, tout autant vocales ou graphiques que chorégraphiques, en contextes rituels amérindiens. Il privilégie les niveaux esthétiques et pragmatiques de l'observation, et ne mobilise leur dimension ontologique que pour soutenir des arguments ponctuels. Masques, aérophones, effigies, trophées, peintures sont ici abordés in situ, du point de vue des effets que leur apparition suscite, mobilisant pour ce faire les théories développées en anthropologie du rituel et en histoire de l'art par des auteurs comme Alfred Gell et Carlo Severi. Le titre de l'ouvrage peut d'ailleurs être lu comme une de ces «chimères » chères à ce dernier, le terme " artifacts " ayant vite fait de se profiler en filigrane derrière " art effects ». Fausto met ainsi en lumière les mécanismes des transformations induites par l'art, pour nous 
emmener au cœur de l'action, dans la minutie des éléments incitant le changement. Il se distancie ce faisant de l'approche perspectiviste prônée par Eduardo Viveiros de Castro, à la fois par l'angle sous lequel les changements sont observés et par les objets analysés eux-mêmes. Les artefacts subissent des transformations, acquièrent en certains contextes une efficacité et affectent les humains. L'agentivité est définie par l'auteur comme "le fait qu'un artefact rituel ait un effet sur ce qui l'entoure, sans que l'on présuppose pour autant qu'il est la source de l'action ou qu'il implique une intention»(p.12). L'agentivité est ainsi explicitement distinguée de l'intention.

3 Les êtres autres qu'humains captivent, notamment du fait de leur capacité à la transformation et à l'intervention dans le monde humain. L'auteur démontre qu'il existe différentes manières de figurer cette aptitude. Il nous convie d'abord au cœur de l'univers parakanã, où les artefacts sont peu nombreux et les occurrences rituelles rares. Le corps, considéré dans la littérature amazonienne comme un objet en constante fabrication, est abordé en tant qu'artefact à partir duquel divers procédés permettent de rendre une "présence-autre " (other presence) tangible. L'énonciation des chants rituels suscite un dédoublement du chanteur, par l'élaboration d'un masque vocal: la voix provient du larynx, ce qui ouvre une zone de doute chez les participants, un obstacle à la compréhension quotidienne. Cette technique dénote la condition duelle du chanteur, à la fois victime et tueur. Cette condition est cependant annihilée lorsque l'ennemi, ou une partie de son corps, est présent sous forme de trophées. Il pouvait s'agir de têtes, d'os utilisés pour la construction d'aérophones ou de dents avec lesquelles des colliers étaient confectionnés.

4 Carlos Fausto établit un continuum, concernant la fabrication des artefacts, allant de l'usage de ressources corporelles à leur production, c'est-à-dire de l'usage de parties du corps prises sur l'ennemi à des substituts de corps produits dans un contexte rituel. La relation avec l'objet est exclusive dans le premier cas, collectivisée dans le second. Après avoir avancé du corps au trophée dans le premier chapitre, l'auteur chemine du trophée aux instruments à vent sacrés au cours du suivant. Il définit les différentes aires géographiques de présence de ces derniers. Le lecteur découvre alors une deuxième facette de cet ouvrage: une méthode comparative - à l'image de celle employée par Claude Lévi-Strauss dans La Voie des masques (1975) - permettant à l'auteur d'interroger la propagation de formes esthétiques. En diverses régions d'Amazonie, des groupes amérindiens pratiquent le complexe des flûtes sacrées, interdit aux femmes. Voir ou entendre l'instrument pourrait leur être fatal. Carlos Fausto prend le temps d'une minutieuse comparaison, analysant les divergences et les convergences des différentes pratiques, pour se concentrer ensuite sur celles des Kuikuro, et ainsi situer leurs flûtes sacrées dans un ensemble d'artefacts musicaux. Là aussi, une présence-autre est convoquée : des mécanismes formels de complexification rituelle engendrent la zone de doute nécessaire. Ces artefacts possèdent le statut particulier d'animal domestique de l'officiant, ce qui permet la capture de la force prédatrice externe, ensuite canalisée par la production de parenté. Les aérophones rituels sont de puissants outils de ce mouvement de capture.

5 Les masques sont équivalents aux aérophones sur de nombreux points. Ils peuvent indexer des classes de personnes - animaux ou ancêtres -, plutôt qu'une relation personnelle. Carlos Fausto initie pour les masques un ample tour d'horizon - qui lui fait cependant perdre en précision - depuis l'Amérique du Nord jusqu'en Amazonie. Il s'interroge sur la façon dont le masque déplace l'attribution de subjectivité, l'évocation 
d'une présence-autre. Le motif de base de tous ces masques est « l'humain qui se trouve à l'intérieur » (p. 125). Le danseur n'est pas possédé par un esprit : il le devient lui-même. Le masque de l'esprit, animé par un humain, contient en fait un humain qui se transforme en non-humain. Les masques ont pour objectif de représenter des êtres « extraordinaires", qui le sont du fait de leurs multiples identités en constante transformation.

6 Après ces analyses mêlant matériaux ethnographiques personnels et bibliographiques, les deux derniers chapitres sont fondés exclusivement sur les données recueillies par l'auteur chez les Kuikuro. Ils concernent l'étude approfondie des relations établies autour de deux effigies anthropomorphiques : celle rudimentaire du rituel du javari et celle très décorée du quarup. Toutes deux représentent des personnes du groupe, décédées, et en passe de devenir des ancêtres. Le javari commémore des chanteurs et des archers de festivals passés, tandis qu'au cours du quarup, chefs et lutteurs sont fêtés.

7 Le javari est abordé en premier lieu. Le rituel dure quinze jours; au cours des deux derniers, il prend une dimension interethnique. Carlos Fausto décrit brièvement les différentes phases du rituel. À travers une approche pragmatique de l'action rituelle, il démontre que le javari est bien plus qu'un rite funéraire, et l'effigie, bien plus qu'un artefact-objet. Cette dernière est un "pivot relationnel», une figure statique et silencieuse contribuant à l'établissement simultané de diverses relations. L'effigie se trouve précisément à l'intersection de deux séries d'identifications. Sur un plan symétrique et horizontal, dans un premier temps, les relations d'affinité apparaissent par l'intervention de cousins croisés : l'une des prérogatives de l'effigie est d'« appeler son cousin ». Une succession de duels verbaux se met effectivement en place à travers la figure interposée de l'effigie, entre cousins croisés de différents villages. Il s'agit d'attaques individuelles, adressées indirectement par l'intermédiaire du lancer d'une javeline sur l'effigie, troisième terme muet et statique d'une interaction dialogique. Une identification mutuelle entre cousins adversaires favorise la construction d'un " je » complexe. Dans un second temps, sur un plan complémentaire et vertical, les valeurs de l'ancestralité sont manifestées par l'identification de l'effigie avec le défunt. Carlos Fausto émet l'hypothèse que ce rituel est le résultat historique d'une "traduction créative » (creative translation, p. 220) d'un rite de trophée. Celle-ci aurait impliqué deux éléments : la substitution du trophée par l'effigie générique, qui permet à la guerre d'être présente sur un mode mineur, exprimée par une pantomime; et l'attribution à l'effigie de l'identité d'une personne défunte du groupe.

8 Ce processus de traduction a eu lieu dans un contexte dans lequel il existait déjà une effigie rituelle, quant à elle grandement décorée : l'effigie du quarup. Contrairement à celle du javari, celle-ci ne semble indexer qu'une unique personne, le chef ou le lutteur commémoré. Chaque effigie figure un individu particulier possédant une biographie propre. Un lutteur, contrairement à un chef, ne peut pas transmettre à sa descendance le privilège d'être fêté au cours d'un quarup. Le rituel produit un état d'incertitude dans lequel le double du défunt est rendu visible à travers l'artefact. Le but est de convoquer sa présence sans pour autant annuler la distance entre présence et représentation. Alors que l'effigie du javari acquiert une multiplicité d'identités simultanées, celle du quarup devient une personne à travers un processus cumulatif, qui prend place dans l'activité rituelle - peinture, chants.

9 Tout au long de son ouvrage, Carlos Fausto convoque des matériaux variés à partir desquels il développe des réflexions passionnantes sur des thèmes annexes : l'altérité, les liens existants entre relations quotidiennes et relations rituelles, ou encore celui de la 
transmission. Ce dernier thème émerge dans toute sa diversité au fil des pages; dans le chapitre 5 portant sur le quarup, nous apprenons par exemple qu'un chef transmet son nom à son petit-fils, que ce dernier en est le « substitut nominal ». Les noms connectent et identifient des personnes de générations alternées. Ce faisant, ils lient des biographies passées et présentes. L'effigie porte le nom du défunt commémoré, et de fait, celui de son petit-fils. Le rituel permet ainsi une reconfiguration entre les vivants, et entre les vivants et les morts. Les divers trophées mentionnés possédaient des destins variés: les têtes étaient souvent détruites ou abandonnées à la fin du cycle rituel; les aérophones en os restaient connectés à leur maître (owner) jusqu'à ce qu'il meure; les colliers de dents étaient quant à eux transmis à travers les générations. Mais c'est surtout la transmission des connaissances qui est abordée. Les flûtes sacrées possédaient par exemple jadis, chez les Kuikuro, un vaste répertoire. Trente-sept pièces sont aujourd'hui jouées. Des questions relatives à la disparition, à la transformation du répertoire sont ainsi abordées. Le lecteur accompagne des discours portant sur l'acceptation, des incorporations culturelles ou rituelles, des disparitions, des emprunts ou des transformations, autant dans les relations internes qu'entre groupes, ou avec des non-Indiens.

Art Effects nous offre en définitive une image empirique de l'Amazonie indigène. Trophées, masques, aérophones y prennent vie au fil des pages. Les exemples sont nombreux et précis lorsqu'il s'agit de comparer des pratiques, approfondis, voire finement disséqués lorsque les données sont issues des terrains de l'auteur. La richesse des données ethnographiques et la pertinence de la contribution théorique en font un ouvrage passionnant et fécond.

\section{BIBLIOGRAPHIE}

LÉVI-STRAUSS Claude

1975 La Voie des masques, Albert Skira (Les sentiers de la création), Genève, 2 vol.

\section{AUTEURS}

CYRIL MENTA

Chercheur associé au Lesc-Erea (UMR 7186), université Paris Nanterre 